\title{
Heating of Nuclei with Energetic Antiprotons
}

\author{
F. Goldenbaum, ${ }^{1}$ W. Bohne, ${ }^{1}$ J. Eades, ${ }^{2}$ T. v. Egidy,${ }^{3}$ P. Figuera, ${ }^{1}$ H. Fuchs,${ }^{1}$ J. Galin, ${ }^{4}$ Ye. S. Golubeva, ${ }^{5}$ K. Gulda, ${ }^{6}$ \\ D. Hilscher, ${ }^{1}$ A. S. Iljinov, ${ }^{5}$ U. Jahnke, ${ }_{1}^{1}$ J. Jastrzebski, ${ }^{6}$ W. Kurcewicz,${ }^{6}$ B. Lott, ${ }^{4}$ M. Morjean, ${ }^{4}$ G. Pausch, ${ }^{7}$ \\ A. Péghaire, ${ }^{4}$ L. Pienkowski, ${ }^{1,6}$ D. Polster, ${ }^{1}$ S. Proschitzki, ${ }^{8}$ B. Quednau, ${ }^{4}$ H. Rossner, ${ }^{1}$ S. Schmid, ${ }^{3}$ W. Schmid,${ }^{3}$ and \\ P. Ziem ${ }^{1}$ \\ ${ }^{1}$ Hahn-Meitner-Institut Berlin, Glienickerstr. 100, D-14109 Berlin, Germany \\ ${ }^{2}$ CERN-PPE, CH-1211-Geneva 21, Switzerland \\ ${ }^{3}$ TU-München, D-85748 Garching, Germany \\ ${ }^{4}$ GANIL (IN2P3-CNRS, DSM-CEA), BP 5027, F-14021 Caen-Cedex, France \\ ${ }^{5}$ INR, Russian Academy of Science, 117312 Moscow, Russia \\ ${ }^{6}$ University of Warsaw, 02-097 Warszawa, Poland \\ ${ }^{7}$ FZ-Rossendorf, D-01314 Dresden, Germany \\ ${ }^{8}$ IPN Orsay, BP 1, F-91406 Orsay-Cedex, France
}

(Received 8 April 1996)

\begin{abstract}
The annihilation of energetic $(1.2 \mathrm{GeV})$ antiprotons is exploited to deposit maximum thermal excitation (up to $1000 \mathrm{MeV}$ ) in massive nuclei $(\mathrm{Cu}, \mathrm{Ho}, \mathrm{Au}$, and $\mathrm{U}$ ) while minimizing the contribution from collective excitation such as rotation, shape distortion, and compression. Excitation energy distributions $d \sigma / d E^{*}$ are deduced from eventwise observation of the whole nuclear evaporation chain with two $4 \pi$ detectors for neutrons and charged particles. The nuclei produced in this way are found to decay predominantly statistically, i.e., by evaporation. [S0031-9007(96)00872-1]
\end{abstract}

PACS numbers: 25.43.+t, 24.60.Dr

The study of such decay modes of very highly excited nuclei as fission, multifragmentation, cracking, and vaporization is presently a major objective in nuclear physics because of its bearing on the lesser-known bulk properties of hot nuclear matter, such as heat capacity, specific heat, viscosity, and phase transitions. Unfortunately, the decay pattern is also very sensitive to the dynamics of the excitation process, especially when collective degrees of freedom like rotation, shape distortion, and compression are strongly induced. These may have to be envisaged in the most often used [1-3] heavy-ion reactions. This ambiguity makes it difficult to correlate the observed decay pattern with either thermally or dynamically induced decay.

In order to minimize the influence of the entrance channel on the decay modes, we have, for the first time, investigated the nuclear excitation following annihilation of energetic antiprotons. Antiprotons annihilate on a single nucleon at the surface of, or even inside the nucleus, thereby producing a pion cloud containing an average of about 5 particles. Because of the high centerof-mass velocity $\left(\beta_{\mathrm{c} . \mathrm{m} .}=0.63\right)$ of this cloud, it is focused forward into the nucleus. Since the pion momenta are comparable to the Fermi momentum of the nucleons in the nucleus, the pions heat the nucleus in a soft radiationlike way [4], probably even softer and more efficient than can be expected in proton- or other lightion-induced spallation reactions, which have also been exploited recently for this purpose [5-7].

Intranuclear cascade (INC) calculations have been found to provide a reasonable description of this mechanism. They predict that the spin remains low (below maximum 25 $)$ and that shape distortion and density compression are negligible [8], in contrast to what is expected in heavy-ion reactions. The reaction time for achievement of equilibrium conditions is only about $30 \mathrm{fm} / c$ or $10^{-22} \mathrm{~s}$ [9], which is much shorter in general than the dynamical period in heavy-ion reactions [10]. This is all the more important at high temperature $(T \approx 6 \mathrm{MeV})$ when the characteristic evaporation time reduces to $t<10^{-22} \mathrm{~s}$, implying little cooling of the compound nucleus during heating.

In this Letter we concentrate on the use of a new method to determine the thermal excitation energy produced with energetic antiprotons. This method is based on the eventwise observation of the whole nuclear evaporation chain, including both neutrons and charged particles. The detailed decay modes of the hot nuclei will be the subject of a forthcoming publication.

The experiment PS208 was carried out at the low-energy antiproton ring (LEAR) at CERN, Geneva. Antiprotons with an energy of $1.2 \mathrm{GeV}$ triggered a scintillator system consisting of a thin $(2 \mathrm{~mm})$ start detector $S 1$ vetoed by an annular detector $S 0$. About $16 \mathrm{~m}$ downstream of $S 1, S 0$, the antiprotons were focused onto ${ }^{\text {nat }} \mathrm{Cu},{ }^{165} \mathrm{Ho},{ }^{197} \mathrm{Au}$, and ${ }^{238} \mathrm{U}$ targets with thicknesses of $1-2 \mathrm{mg} / \mathrm{cm}^{2}$.

The reaction products induced by energetic $\bar{p}$ were detected by means of two $4 \pi$ detectors surrounding the target: the so-called Berlin Neutron Ball (BNB) containing at its center the Berlin Silicon Ball (BSiB). The BNB [11] is a spherical tank with an outer diameter of $140 \mathrm{~cm}$ and a scintillator volume of $1500 \mathrm{l}$, housing a reaction chamber of $40 \mathrm{~cm}$ diameter at the center of which the targets were located. This detector was 
mainly used for counting evaporationlike neutrons in each reaction. For these low-energy neutrons, its efficiency was typically $\epsilon \sim(83-85) \%$. For cascade neutrons of higher energy $(30-50 \mathrm{MeV})$, the efficiency decreases to the $(40-25) \%$ level [11], which means that the detector is rather transparent to neutrons from pre-equilibrium processes. The master trigger for the acquisition system was a coincidence of $S 1 \wedge \overline{S 0}$ and the prompt light signal of the BNB, with the threshold set to $\approx 10 \mathrm{MeV}$. The prompt light comes from all kinds of reaction products, de-excitation $\gamma$ rays, recoil protons from neutrons slowing down in the scintillator, but most of all from high-energy charged pions from the primary annihilation process.

Light-charged particles (LCP: $\mathrm{H}+\mathrm{He}$ isotopes), intermediate mass fragments (IMF), and fission fragments (FF) were detected by the BSiB [12] composed of 158 independent silicon detectors $(500 \mu \mathrm{m}$ thick) forming a $20 \mathrm{~cm}$ diameter sphere. These detectors covered an active zone of about $90 \%$ of $4 \pi$. Because of absorption of LCP's in the target, the detection efficiency for LCP's decreases further to about (79-84)\%, depending on the $Z$ of the particle. For each detected particle the time of flight (TOF) as well as energy $E$ was measured. Charged particles $(\mathrm{CP}: \mathrm{H}+\mathrm{He}+\mathrm{IMF}+\mathrm{FF})$ were identified by means of TOF versus $E$ correlations with a lower detection threshold of less than $1 \mathrm{MeV}$.

The combined information from the two $4 \pi$ detectors is shown in the upper panel of Fig. 1 as the correlation between observed multiplicities for neutrons and CP's from reactions on $\mathrm{Cu}, \mathrm{Ho}, \mathrm{Au}$, and $\mathrm{U}$. As a general
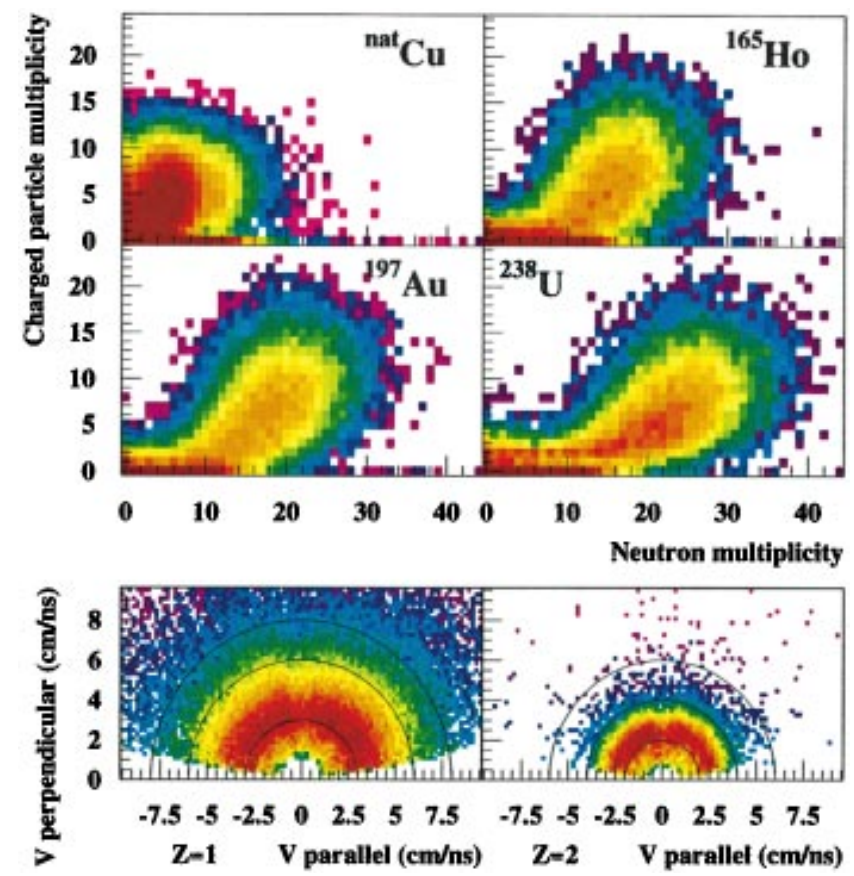

FIG. 1(color). Top panels: Correlation of neutron multiplicity versus $\mathrm{CP}$ multiplicity for $1.2 \mathrm{GeV} \bar{p}$ induced reactions on $\mathrm{Cu}$, $\mathrm{Ho}, \mathrm{Au}$, and $\mathrm{U}$, corrected for background but not for efficiency. Bottom panels: Galilean-invariant velocity plot for evaporated $Z=1$ and $Z=2$ particles for $1.2 \mathrm{GeV} \bar{p}+\mathrm{Au}$. tendency, we note an increase of both, $M_{n}$ and $M_{\mathrm{CP}}$, with increasing $A$ and $Z$ of the target. This is mainly due to the fact that larger target nuclei incorporate more energy from the pionic system and have lower particle separation energies than do lighter ones. Also, at lower excitations for heavy nuclei $(\mathrm{Au}, \mathrm{U}$ relative to $\mathrm{Cu}, \mathrm{Ho}$ ) emission of neutrons is strongly favored over that of LCP's, and at higher excitations, when comparing Au to $\mathrm{U}$, we observe once more a shift of the measured distributions toward larger neutron multiplicity as a result of a further reduction of neutron separation energies.

The method employed to determine the thermal excitation energy relies on the basic property of hot nuclei of de-excitation by evaporation of light particles (LP: neutrons + light charged particles), thereby carrying off some 10 to $20 \mathrm{MeV}$ of excitation energy per particle, approximately equally divided between binding and kinetic energies. This evaporation process is almost perfectly described by the many existing statistical model codes. A prerequisite for the applicability of these models is that the source is equilibrated by the time the emission starts. In order to account for this condition, we cut off at $24 \mathrm{MeV}$ the relatively small (typically 15\%) contribution at higher energy from the energy spectra of the detected LCP's having a different slope and verify the isotropic emission of the remaining evaporation part. The fact that the Galilean-invariant velocity distribution presented in the lower panel of Fig. 1 follows circles centered in the origin of the velocity plane clearly demonstrates that $\mathrm{H}$ and $\mathrm{He}$ particles are isotropically emitted from an equilibrated thermalized system, which is nearly at rest due to the small recoil from the reaction. It is also noteworthy that the most energetic $Z=1$ particles are not registered at all due to the lower energy threshold of the $E$ detectors. For neutrons, however, we have only indirect information on the kinetic energy on account of the variability in detection efficiencies pointed out above.

In order to infer for each reaction event the induced thermal excitation $E^{*}$, we use the sum of all registered light particles $M_{\mathrm{LP}}$, and associate with it (after correction for efficiency) the excitation energy $E^{*}$. For the calculation of the relation $M_{\mathrm{LP}}\left(E^{*}\right)$, we employ here a slightly extended version [13] of the statistical model code GEMINI [14], because this code also allows for IMF emission. Figure 2 demonstrates the sensitivity of the assignment of $E^{*}$ to $M_{\mathrm{LP}}$, and also points out its advantage over allocating $E^{*}$ from the neutron multiplicity $M_{n}$ alone. The upper two panels show, as an example, the experimental multiplicity distributions $d \sigma / d M$ for $1.2 \mathrm{GeV}$ $\bar{p}+\mathrm{Au}$ as dotted curves for all LP (a) and for neutrons only (b). Calculated multiplicity distributions $M_{\mathrm{LP}}\left(E^{*}\right)$ and $M_{n}\left(E^{*}\right)$ have been included for a set of fixed values $E^{*}=100,200,300,400,600,800$, and $1000 \mathrm{MeV}$. These $M_{\mathrm{LP}}$ distributions are very well separated from each other, showing the strong correlation between the two quantities $M_{\mathrm{LP}}$ and $E^{*}$. The same comparison for neutrons only is much less favorable: here the $M_{n}$ distributions already start 

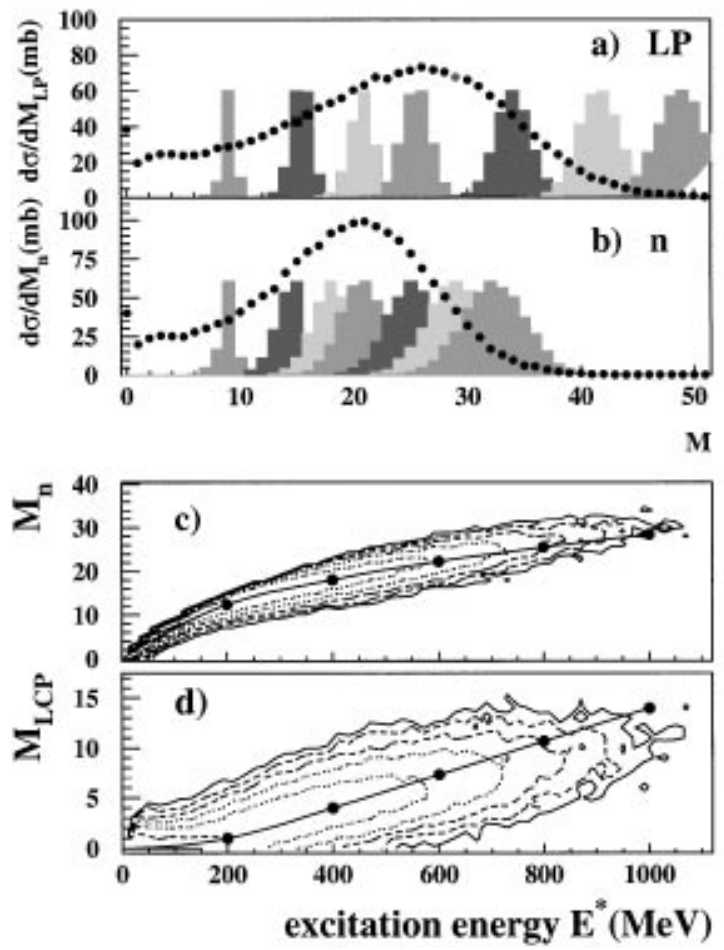

FIG. 2. Upper two panels: Measured multiplicity distributions (O) $d \sigma / d M_{\mathrm{LP}}$ (a) and $d \sigma / d M_{n}$ (b) for $1.2 \mathrm{GeV} \bar{p}+\mathrm{Au}$. The shaded histograms are calculated multiplicity distributions for fixed values of $E^{*}=100,200,300,400,600,800$, and $1000 \mathrm{MeV}$ (from left to right). Lower two panels: Contour diagrams of experimental event distributions for $M_{n}$ (c) and $M_{\mathrm{LCP}}(\mathrm{d})$ as a function of $E^{*}$ compared with calculated average multiplicities (dots connected by a line). The intensity change between two contour lines is a factor of 3 .

overlapping at low excitation. The FWHM of these calculated multiplicity distributions can be translated into an energy width $\Delta E^{*}$ defining an energy resolution $\Delta E^{*} / E^{*}$. By increasing the excitation energy from 150 to $1000 \mathrm{MeV}$ for $\mathrm{Au}$, the thus defined energy resolution decreases from $50 \%$ to $11 \%$ if deduced from $M_{\mathrm{LCP}}$, it increases from $12 \%$ to $23 \%$ for $M_{n}$, and assumes a constant value of $7 \%$ for $M_{\mathrm{LP}}$. We conclude that $M_{\mathrm{LP}}$ is indeed a reliable observation for $E^{*}$ up to $1 \mathrm{GeV}$ or more but that the observation of $M_{n}[13,15]$ and of $M_{\mathrm{LCP}}$ [2] alone (which have been applied before for this purpose) is less sensitive to and the resolution depends strongly on the excitation energy. Since the next best choice are LCP's, at least for high excitation energies, we have also reconstructed the excitation energy distributions by using $M_{\mathrm{LCP}}$ only. This comparison agrees for heavy nuclei quite well with the LP method, but deviates for $\mathrm{Cu}$ as will be discussed below.

It is also shown in the lower part of Fig. 2 for Au that the model predictions (continuous lines) fit closely the ridge of the event distributions as a function of $M_{n}$ (c) and $M_{\mathrm{LCP}}(\mathrm{d})$, showing that the sharing between $n$ and LCP is also well accounted for on the average.

There are some uncertainties in the choice of the model parameters which have to be considered because of their relevance for the reliability of the deduced value $E^{*}$. For instance, in the calculation we take into account that in the course of the fast cascade phase an original ${ }^{238} \mathrm{U}$ nucleus loses mass and charge with increasing $E^{*}$ up to ${ }^{214} \mathrm{At}$, as is suggested by the INC calculation for $E^{*}=1 \mathrm{GeV}$. For this extreme $E^{*}$ the respective evaporation chain releases about 3 neutrons less, but $1 \mathrm{CP}$ more than it would have for an intact ${ }^{238} \mathrm{U}$ nucleus. Also, a variation of the level density parameter and the spin within reasonable limits (from $a=A / 10$ to $A / 8.5$ or $A / 13$ and from $l=0 \hbar$ to $25 \hbar$ ) results at best in a variation of $M_{\mathrm{LP}}$ by 3 to 4 units at the highest excitation, which corresponds to an uncertainty for $E^{*}$ of about $\pm 10 \%$. The results from other statistical model codes, JULIAN [16] or from Ref. [17], are once more consistent with the ones from GEMINI within these limits. Incidentally, the results from different codes agree much better in the sum multiplicity $M_{\mathrm{LP}}$ than in the ratio $M_{\mathrm{CP}} / M_{n}$. Finally, it is worth noting that a result of statistical model calculations is that fission or breakup of the nucleus into three or more heavy fragments at any stage along the de-excitation chain does not alter $M_{\mathrm{LP}}$ or $E^{*}$ by more than $10 \%$. Therefore, this method is not subject to specific splitting modes of the hot nucleus.

Using the method described above, we have been able to deduce excitation energy distributions and absolute differential cross sections $d \sigma / d E^{*}$ following $\bar{p}$ annihilation for the first time. These are shown as dotted curves in Fig. 3. Also included in Fig. 3 are the pertinent INC calculations [8] as histograms. For the heavy nuclei Ho, Au, and $U$, we note a satisfactory agreement between experimental and model distributions both in shape and in absolute values. For $\mathrm{Cu}$, however, we observe a considerable discrepancy near the maximum close to $E^{*}=150 \mathrm{MeV}$. For this relatively light nucleus the experimental reconstruction of the $E^{*}$ distribution from the multiplicity $M_{\mathrm{LP}}$ of all light particles might be less reliable because of the difficulty in discriminating between evaporative and directly emitted neutrons and in subtracting the very few (1 or 2) additional neutrons from pion-induced reactions in the scintillator liquid of BNB. This has the tendency to transfer cross section from low $E^{*}$ to intermediate $E^{*}$. Since the relative contribution of these two effects is more important for lighter target nuclei, we show for $\mathrm{Cu}$ in Fig. 3 (star symbols) also the $E^{*}$ distribution deduced from $M_{\mathrm{LCP}}$ alone which seems to agree somewhat better with the INC calculation.

All four $d \sigma / d E^{*}$ distributions are dominated by a broad distribution which shifts to higher $E^{*}$ for the heavier nuclei. At very low $E^{*}$ the intensity increases considerably, which we assign to peripheral reactions. More important with respect to properties of hot nuclear matter are the high energy tails of the data. The $\bar{p}$ interaction with uranium, for instance, leads for more than $12 \%$ of the reaction cross section to thermal energies in excess of $600 \mathrm{MeV}$, i.e., to temperatures larger than $5.2 \mathrm{MeV}$ (with $a=A / 10$ ). In the extreme tails of the distributions even energies as high as $1 \mathrm{GeV}$ are reached, which could not 


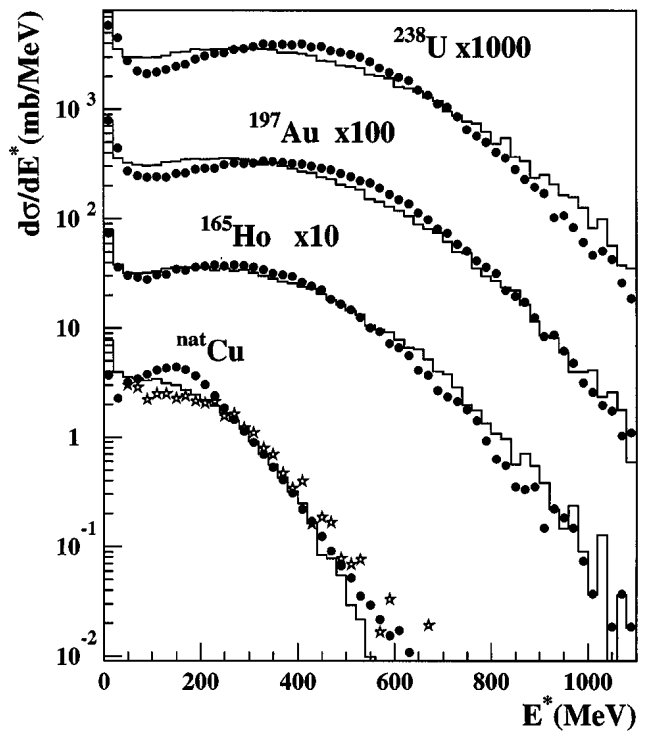

FIG. 3. Distribution of excitation energy $d \sigma / d E^{*}$ deduced from $M_{\mathrm{LP}}()_{)}$or $M_{\mathrm{LCP}}(\star)$ compared with the INC model (histogram) for $\bar{p}(1.22 \mathrm{GeV})+\mathrm{U}, \mathrm{Au}, \mathrm{Ho}$, and $\mathrm{Cu}$.

be obtained [7] with protons of still higher incident energy $(2 \mathrm{GeV})$. This finding verifies the expectation [9] that antiprotons are more efficient in heating nuclei than protons.

Table I collects the parameters deduced from the $E^{*}$ distributions in Fig. 3. These parameters are the mean excitation energy $\left\langle E^{*}\right\rangle$, the mean excitation energy per nucleon $\left\langle E^{*} / A\right\rangle$, and the maximum excitation energies $E_{\max }^{*}$ and $E_{\max }^{*} / A$, defined by the somewhat arbitrary criterion that they are associated to the upper $1 \%$ of the excitation energy distribution. The average $\left\langle E^{*}\right\rangle$ from $\bar{p}$ annihilation in flight increases from $\mathrm{Cu}$ to $\mathrm{U}$ almost linearly with $A$ in accordance with INC model predictions. However, when converted to $\left\langle E^{*} / A\right\rangle$, this tendency is inverted: the lighter nucleus receives more excitation energy per nucleon or equivalently higher temperature. As to the maximum excitation energy, $E_{\max }^{*}$ in Table I, we note that for $U$ as much as $30 \%$ of the totally available energy $(3.1 \mathrm{GeV})$ is converted to intrinsic excitation, but still $E_{\max }^{*} / A=4.3$ is below the expected [17] onset of

TABLE I. Target dependence of $\left\langle E^{*}\right\rangle,\left\langle E^{*} / A\right\rangle$, and measured reaction cross section $\sigma_{\text {reac }}$ as well as geometrical cross section $\sigma_{\text {geo }}\left(r_{0}=1.38 \mathrm{fm}\right)$. Numbers in parentheses show the maximum measured $E^{*}$ or $E^{*} / A$ corresponding to $1 \%$ of $\sigma_{\text {reac }}$, assuming for $A=A_{\text {target }}-\Delta A_{\text {INC }}$ with $\Delta A_{\text {INC }}$ being the calculated mass loss in the INC stage.

\begin{tabular}{|c|c|c|c|c|}
\hline${ }_{Z}^{A} X$ & $\begin{array}{c}\left\langle E^{*}\right\rangle\left\langle E_{\max }^{*}\right\rangle \\
(\mathrm{MeV})\end{array}$ & $\begin{array}{c}\left\langle E^{*} / A\right\rangle\left(E_{\max }^{*} / A\right) \\
(\mathrm{MeV})\end{array}$ & $\begin{array}{l}\sigma_{\text {reac }} \\
(\mathrm{mb})\end{array}$ & $\begin{array}{l}\sigma_{\text {geo }} \\
(\mathrm{mb})\end{array}$ \\
\hline${ }^{\text {nat }} \mathrm{Cu}$ & $144 \pm 20(516)$ & $2.53(11.3)$ & $973 \pm 80$ & 950 \\
\hline${ }^{165} \mathrm{Ho}$ & $269 \pm 30(780)$ & $1.73(5.4)$ & $1817 \pm 95$ & 1800 \\
\hline${ }^{197} \mathrm{Au}$ & $309 \pm 30(880)$ & $1.65(5.0)$ & $1985 \pm 110$ & 2025 \\
\hline${ }^{238} \mathrm{U}$ & $348 \pm 40(940)$ & $1.52(4.3)$ & $2220 \pm 130$ & 2290 \\
\hline
\end{tabular}

nonevaporative emission of IMF's. The observed mean IMF multiplicities for all four reactions are below 1.2 up to the highest excitation energies which can be fully explained by evaporation.

By integrating $d \sigma / d E^{*}$ we obtain the reaction cross sections $\sigma_{\text {reac }}$ (Table I) for an inelasticity larger than about $10 \mathrm{MeV}$. The values so obtained compare rather well with a geometrical cross section $\sigma_{\text {geo }}$ calculated with $r_{0}=1.38 \mathrm{fm}$. The radius parameter is thus slightly larger than the standard value, which indicates that annihilation can occur already in the low density periphery of the nucleus.

In summary, our studies have shown energetic antiprotons to be a promising tool to create high thermal excitation in massive nuclei with minimum stimulation of collective motion. Excitation energy spectra for $1.2 \mathrm{GeV}$ $\bar{p}+\mathrm{Cu}, \mathrm{Ho}, \mathrm{Au}$, and $\mathrm{U}$ extend with appreciable cross section ( $1 \%$ of $\sigma_{\text {reac }}$ ) up to $500 \mathrm{MeV}$ for $\mathrm{Cu}$ and as far as about $1000 \mathrm{MeV}$ for $\mathrm{U}$. They are in satisfactory agreement with predictions from the INC model.

The excitation energy distributions are deduced from eventwise observation of the whole nuclear de-excitation chain, a method which became feasible by the combined application of two $4 \pi$ detectors for LCP's and for neutrons. The precision of this new method of determining thermal excitations for heavy nuclei is estimated to be about $\pm 10 \%$ up to $E^{*}=1 \mathrm{GeV}$, with the benefit of minimum bias from reaction models. The decay of hot nuclei produced with energetic antiprotons and a minimum of collective excitation such as rotation, compression, and shape distortion exhibit essentially a statistical decay via evaporation up to 5 or even $11 \mathrm{MeV} /$ nucleon of thermal excitation energy.

We are very much indebted to the LEAR staff at CERN for the good beam quality and also thank E. Widmann and M. Doser for continuous support.

[1] J. Pochodzalla et al., Phys. Rev. Lett. 75, 1040 (1995).

[2] D. R. Bowman et al., Phys. Rev. Lett. 67, 1527 (1991).

[3] J. Tõke et al., Phys. Rev. Lett. 75, 2920 (1995).

[4] D. Polster et al., Phys. Rev. C 51, 1167 (1995).

[5] V. Lips et al., Phys. Rev. Lett. 72, 1604 (1994).

[6] K. Kwiatkowski et al., Phys. Rev. Lett. 74, 3756 (1995).

[7] L. Pienkowski et al., Phys. Lett. B 336, 147 (1994).

[8] Ye.S. Golubeva et al., Nucl. Phys. A483, 539 (1988); A. S. Iljinov et al., Nucl. Phys. A382, 378 (1982).

[9] J. Cugnon et al., Nucl. Phys. A470, 558 (1987).

[10] W. Bauer et al., Phys. Rev. Lett. 69, 1888 (1992).

[11] J. Galin and U. Jahnke, J. Phys. G: Nucl. Part. Phys. 20, 1105 (1994).

[12] P. Figuera et al., Z. Phys. A352, 315 (1995).

[13] B. Lott et al., Z. Phys. A346, 201 (1993).

[14] R. J. Charity et al., Nucl. Phys. A483, 371 (1988).

[15] D. X. Jiang et al., Nucl. Phys. A503, 560 (1989).

[16] H. Rossner et al., Phys. Rev. C 40, 2629 (1989).

[17] W. Friedman, Phys. Lett. B 242, 309 (1990). 